\title{
EFDS-Workshop
}

\section{Nasschemische Reinigung optimal beherrschen}

\author{
Am 24. Januar 2013 veranstaltete die Europäische Forschungsgesellschaft Dünne Schichten (EFDS) in \\ Kooperation mit dem Fraunhofer-Institut für Elektronenstrahl- und Plasmatechnik, dem Fachverband \\ industrielle Teilereinigung sowie dem ZIM-Netzwerkprojekt „Nasschemische Reinigung für die \\ Oberflächentechnik - NassRein“ den jährlichen Workshop „Nasschemische Reinigung - Optimal \\ beherrschen!“. Schwerpunkt der 4. Veranstaltung war das Thema „Effizienter Einsatz der Ressourcen“.
}

n Dresden kamen 58 Teilnehmer zusammen und nutzten den Workshop zum intensiven Informations- und Erfahrungsaustausch. Im Mittelpunkt der Vorträge standen Kriterien für eine wirtschaftliche und qualitätssichernde Prozessführung

\section{Prozessführung auf höherem} Niveau erforderlich

Zum Workshopauftakt wurden die Ergebnisse einer Studie zum Stand der Technik innerhalb des Netzwerkes NassRein sowie Erfahrungen eines Messtechnik-Spezialisten zur Prozessoptimierung durch Badüberwachung und Sauberkeitskontrolle präsentiert. Notwendig ist ein neues Niveau der Prozessführung in der industriellen Teilereinigung. Durch Qualitätslenkung, das heißt „Qualität erzeugen statt erprüfen“ ist die Sauberkeit von Bauteilen stabil zu sichern. Dies erfordert Forschungsarbeiten zu verfahrenstechnischen Grundlagen für wissensbasierte Prozessführungskonzepte sowie zu neuen Verfahren und Techniken für die Prozessüberwachung und Teilekontrolle.

Der Vortrag „Einflussfaktoren bei der Spritzreinigung von Maschinen- oberflächen “verdeutlichte grundlegende Zusammenhänge zwischen Betriebsparametern und Reinigungswirkung und zeigte Optimierungspotenziale auf.

Zum Thema „Ultraschall-unterstützte wässrige Reinigung von oberflächenveredelten medizinischen Implantaten" wurden grundlegende Untersuchungsergebnisse über Einfluss und Wirkung des Ultraschalls vorgestellt, insbesondere der Parameter Ultraschallfrequenz sowie -leistung und Kavitationsrauschpegel.

In einem weiteren Vortrag wurde die Prozessoptimierung der nasschemischen Reinigung durch Kontrolle der Reinigerkonzentration und Badverschmutzung beleuchtet. Dabei wurde der Zusammenhang von Prozessüberwachung, Prozesssicherheit und ressourceneffizienter Prozessführung eindrucksvoll dargestellt.

Ein Vertreter eines Reinigungsmittelherstellers referierte über Reinigeroptimierung in der wässrigen Teilereinigung und Vorbehandlung und mahnte eine frühzeitige Berücksichtigung und Einbeziehung der Teilereinigung in den gesamten Wertschöpfungsprozess der Teileherstellung an.

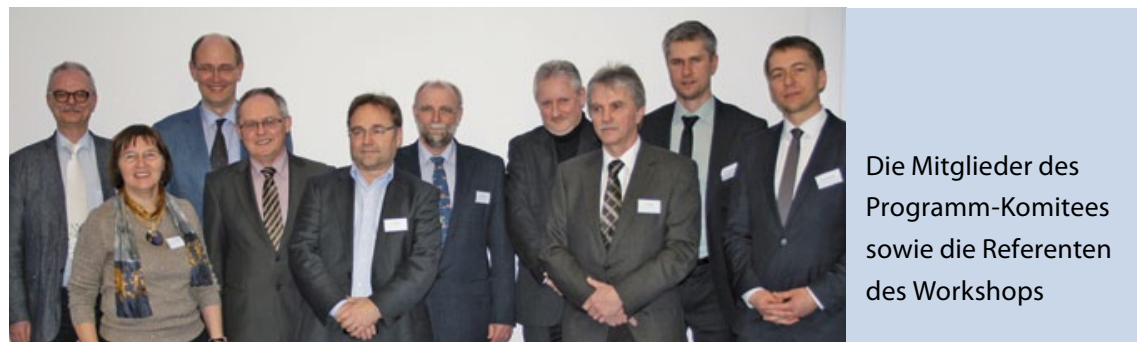

Aus der Sicht eines Anlagenbauers verdeutlichte ein Vortragender die Wirkzusammenhänge von Reinigen, Spülen, Konservieren und Trocknen bei der nasschemischen Reinigung. Er wies besonders darauf hin, dass die Effizienzforderung bei der Teileherstellung keinen Bogen um die Reinigung machen darf. Die ganzheitliche Prozessbetrachtung führt zu effektiveren Abläufen und damit einhergehenden Kostenreduzierungen.

Aufgezeigt wurde ferner die Notwendigkeit der Badpflege, zum Beispiel durch Ultrafiltration, und der Spülwasseraufbereitung. Anhand von Beispielen kundenspezifischer Anlagen zur Kreislaufführung wurde das Potenzial zur Einsparung von Wasser, Energie, Chemie und Kosten verdeutlicht.

\section{Weiterer Forschungsbedarf}

In Summe zeigten die Erfahrungsberichte des Workshops, dass hinsichtlich Prozesssicherheit und stabiler Qualität in der nasschemischen Reinigung bei effizientem Einsatz der Ressourcen bereits wesentliche Verbesserungen erreicht wurden. Gleichzeitig wurde Optimierungspozential aufgezeigt, was sowohl grundlegende Forschung zur Prozessführung als auch bauteil-angepasste Optimierungen erfordert. I

\section{Dr. Ludwig van Loyen}

EFDS Dresden, Netzwerkmanager von "NassRein", www.efds.org/nassrein, vanloyen@efds.org 